\title{
A theoretical and aporetic framework on sexual murder and the description of a clinical case
}

\section{Un quadro teorico e aporetico sull'omicidio sessuale e la descrizione di un caso clinico}

\section{Un marco teórico y aporético sobre el asesinato sexual y la descripción de un caso clínico}

\author{
Francesca Vitale \\ Criminologa clinica ex art. 80, Casa di Reclusione di Milano-Opera; Dottoressa di ricerca in Etica e Antropologia; \\ Researcher and Clinic (Association for the Treatment of Sexual Abusers); Mediatrice umanistica di conflitti; \\ Docente a contratto per i Corsi di Laurea in Filosofia e Scienze Filosofiche, Università degli Studi di Milano, Italy
}

\begin{abstract}
Homicide represents a very complex criminal event. While there is a voluminous international literature on the (non-sexual) murder phenomenon, the scientific production on the topic of sexual homicide appears incomplete. This is imputable to a series of problems that hinder the advancement of specialist scientific knowledge. The present article aims, first of all, to describe the problems that go across the activity of research in the field of sexual homicide and then, to make a review of the main theoretical models developed to understand this type of crime. Finally, a case of Italian crime reports is analyzed through a criminological interpretation emerging from recent studies on sexual homicide.
\end{abstract}

\section{RIASSUNTO}

L'omicidio rappresenta un evento delittuoso assai complesso. Mentre esiste una voluminosa letteratura internazionale sul fenomeno dell'omicidio (non sessuale), la produzione scientifica in materia di omicidio sessuale appare lacunosa. Ciò è attribuibile a una serie di problemi che ostacolano l'avanzamento della conoscenza scientifica sul tema. Il presente articolo mira, anzitutto, a descrivere i problemi che attraversano l'attività di ricerca nel campo dell'omicidio sessuale e, poi, a effettuare una rassegna dei principali modelli teorici elaborati per comprendere tale tipologia omicidiaria. Infine, si analizza un caso di cronaca nera italiana attraverso una chiave di lettura di stampo criminologico emergente da recenti studi sull'omicidio sessuale.

\section{RESUMEN}

El homicidio representa un evento criminal muy complejo. Si bien existe una voluminosa literatura internacional sobre el fenómeno del homicidio (no sexual), la producción científica sobre el tema del homicidio sexual parece deficiente. Esto es atribuible a una serie de problemas que obstaculizan el avance de los progresos de los conocimientos científicos sobre el tema. El objetivo de este artículo es, en primer lugar, describir los problemas que atraviesan la actividad investigadora en el campo del homicidio sexual y, a continuación, hacer una revisión de los principales modelos teóricos desarrollados para comprender este tipo de homicidios. Por último, un caso de crónica criminal italiana se analiza a través de una clave de lectura criminológica que surge de los recientes estudios sobre homicidio sexual.

\section{Introduzione}

L'omicidio è l'atto di soppressione di una o di più vite umane. Per comprenderne la genesi, sono state formulate varie teorie esplicative di matrice sociologica, neurobiologica e psicologica (Lorenz, 1963; Wolfgang \& Ferracuti, 1967; Sutherland \& Cressey, 1982; Berkowitz, 1989; Palermo, 2010; Raine, 2013; Menninger, 1963; Archambault \& Mormont, 1998; Palermo \& Mastronardi, 2005), ma nessuna di esse appare in grado di fornire, da sola, una spiegazione esaustiva di tale fenomeno delittuoso. La comune accezione di "omicidio", inoltre, incorpora diverse realtà tipologiche sia sotto il profilo giuridico sia sotto il profilo criminologico (Merzagora Betsos, 2006; Rossi \& Zappalà, 2005; Palermo \& Mastronardi, 2012). Mentre esiste una volumi- nosa letteratura internazionale sul fenomeno dell'omicidio (non sessuale), la produzione scientifica in materia di omicidio sessuale appare lacunosa.

\section{Aspetti problematici}

La scarsità di studi scientifici in tema di omicidio sessuale è in gran parte attribuibile a una serie di problemi che ostacolano la ricerca scientifica e, di conseguenza, la comprensione di questo tipo di delitto.

Schlesinger (2004) individua i seguenti problemi:

1. nella comunità scientifica internazionale non vi è accordo unanime sulla definizione di "omicidio sessuale"; 
2. molti omicidi apparentemente a sfondo sessuale sono in realtà delitti privi di movente sessuale;

3. molti omicidi apparentemente privi di movente sessuale sono in realtà delitti a sfondo sessuale;

4. la distinzione tra "omicidio sessuale" e "omicidio associato a comportamento sessuale" è confusiva;

5. l'assenza di dati statistici ufficiali a livello nazionale o locale volti a registrare l'incidenza dell'omicidio sessuale;

6. la presenza di ostacoli di natura pratica che impediscono lo svolgimento dell'attività di ricerca sugli autori di reato.

Problema 1. Ressler et al. (1988, pag. 1) definiscono l'omicidio sessuale come "l'uccisione di una persona in un contesto di potere, sessualità e brutalità". Poiché quasi tutti gli omicidi implicano un certo grado di potere e di brutalità, in che modo la presenza di questi elementi diventa criterio per distinguere un omicidio sessuale da un omicidio non sessuale? E qual è il significato univoco del termine "sessualità" adottato in questa definizione? Il termine fa riferimento al comportamento sessuale vero e proprio? Per far sì che l'uccisione si configuri come omicidio sessuale, deve esserci stato un rapporto sessuale, un tentativo di rapporto sessuale, un desiderio di rapporto sessuale, o è sufficiente si sia manifestata una sensazione interna di eccitazione? Come è possibile in sede di indagine determinare se la sensazione provata dall'offensore sia stata di eccitazione sessuale o di sovraccarico emotivo derivante dalla commissione del crimine e/o dal rischio di essere arrestato? Questi sono solo alcuni degli interrogativi che rispecchiano le incertezze teoriche gravanti sul problema definitorio dell'omicidio sessuale.
Unitamente alle differenti definizioni (Tab. 1), sono stati impiegati termini assai diversi per descrivere l'omicidio sessuale, tra cui "Lust murder" (Krafft-Ebing, 1886), "Sadistic lust murder" (De River, 1958), "Sadistic murder" (Brittain, 1970), "Lust killing" (Malmquist, 1996), "Erotophonophilia" (Money, 1990). A volte lo stesso termine, come "Lust murder", è utilizzato con accezioni diverse (Hazelwood \& Douglas, 1980). Sebbene le differenze terminologiche e definitorie rendano difficile la divulgazione e l'utilizzazione dei risultati ottenuti dall'attività di ricerca, esse sono da ritenersi uno stadio di sviluppo normale e, perciò, prevedibile, del processo di ricerca, limitato alle fasi iniziali.

Problema 2. Alcuni casi di omicidio, per esempio quelli seriali, presentano dei problemi peculiari rispetto ai casi tradizionali di omicidio, di cui bisogna tenere conto in fase di investigazione. Uno dei problemi principali riguarda la corretta valutazione della cosiddetta "reciprocità letale", cioè "l'inquadramento, nel suo significato reale, di ogni elemento isolato nella scena del crimine, dei movimenti dell'assassino e della vittima, di cosa abbia collegato la vittima all'assassino e del motivo per cui l'assassino abbia ucciso proprio quella persona" (Pais \& Perrotta, 2015, pag. 168). Dunque, sebbene in alcuni casi la presenza di elementi sessuali sulla scena del crimine, come la mutilazione aggressiva e/o la mutilazione necromanica, sembri legittimare l'ipotesi di omicidio sessuale, ad un esame più attento tale ipotesi potrebbe essere smentita.

Il caso di Richard Trenton Chase, soprannominato il "Vampiro di Sacramento" ne è un esempio. Chase credeva che il suo sangue stesse evaporando e che l'unico modo per restare in

Tab. 1. L'omicidio sessuale: termini, descrizioni, definizioni (Schlesinger, 2004, pag. 22).

\begin{tabular}{|c|c|c|c|}
\hline Autore & Anno & Termine & Descrizione/Definizione \\
\hline Kraft-Ebing & 1886 & Lust murder & $\begin{array}{l}\text { "Il legame tra lussuria e desiderio di uccidere" (p. 62); "Il crimine } \\
\text { sadico è di per sé l'equivalente del coito" (p. 64). }\end{array}$ \\
\hline De River & 1958 & Sadistic lust murder & $\begin{array}{l}\text { "Dopo aver ucciso la vittima, l'assassino tortura, taglia, mutila o } \\
\text { squarcia la vittima... [infierisce] su parti [del corpo] che hanno per } \\
\text { lui un forte significato sessuale e che fungono da stimolo sessuale" } \\
\text { (p. 40). }\end{array}$ \\
\hline Brittain & 1970 & Sadistic murder & $\begin{array}{l}\text { Non offre una definizione specifica, ma fornisce una descrizione di } \\
\text { nove pagine dei tratti di personalità e delle caratteristiche degli } \\
\text { assassini sadici, distinguendo l'omicidio sadico dall'omicidio sessuale. }\end{array}$ \\
\hline Hazelwood, Douglas & 1980 & Lust murder & $\begin{array}{l}\text { "Si distingue dall'omicidio sadico per perché implica una mutilazione } \\
\text { aggressiva o lo spostamento dei seni, del retto o dei genitali" (p. 1). }\end{array}$ \\
\hline MacDonald & 1986 & Sex murder & $\begin{array}{l}\text { "L'elemento sessuale è chiaramente evidente... oppure a volte uno } \\
\text { studio più approfondito rivelerà che il conflitto sessuale è alla base } \\
\text { dell'atto di aggressione" (p. 164). }\end{array}$ \\
\hline Ressler, Burgess, Douglas & 1988 & Sexual homicide & $\begin{array}{l}\text { "Omicidio con prove o rilevazioni indicanti che l'omicidio era di } \\
\text { natura sessuale" (p. xiii). }\end{array}$ \\
\hline Money & 1990 & Erotophonophilia & Omicidio associato al sadismo sessuale come definito nel dsm (p. 26). \\
\hline Douglas et al. & 1992 & Sexual homicide & $\begin{array}{l}\text { "Implica un elemento sessuale (attività) alla base della sequenza di } \\
\text { atti che portano alla morte" (p. 123). }\end{array}$ \\
\hline Douglas et al. & 1992 & Sadistic murder & $\begin{array}{l}\text { "L'aggressore trae massima soddisfazione dalla reazione della vittima } \\
\text { alla tortura" (p. 136). }\end{array}$ \\
\hline Grubin & 1994 & Sexual murder & $\begin{array}{l}\text { "L'uccisione può anche essere strettamente collegata all'elemento } \\
\text { sessuale di una aggressione... il controllo esercitato dall'aggressore } \\
\text { sulla vittima, il dolore e l'umiliazione di quest'ultima diventano } \\
\text { basilari per l'eccitazione sessuale dello stesso" (p. 624). }\end{array}$ \\
\hline Malmquist & 1996 & Lust killing & $\begin{array}{l}\text { "L'obiettivo primario è uccidere la vittima come parte di un attacco } \\
\text { ritualizzato... la motivazione... è la messa in atto di una fantasia che } \\
\text { lo/la ossessiona da tempo" (p. 295). }\end{array}$ \\
\hline
\end{tabular}


vita fosse bere il sangue di altri esseri viventi. In preda ad allucinazioni uditive che gli ordinano di uccidere e in risposta alla credenza delirante di dover bere il sangue delle vittime per restare in vita, Chase uccide sei persone. Secondo Ressler \& Shachtman (1992) Chase incarna il profilo del serial killer disorganizzato, perché alcune delle sue vittime sono state sottoposte a mutilazioni genitali. Tuttavia, Chase uccide anche un neonato di 22 mesi sparandogli in testa, uomini e animali; comportamento atipico per un omicida sessuale. Negli omicidi commessi da Chase sono inequivocabilmente presenti elementi sessuali, ma la violenza omicida sembra essersi innescata in risposta a una forma di delirio psicotico severo. Senza la progressione dello stato psicotico è quantomeno dubbio che Chase avrebbe ucciso.

Problema 3. Molte aggressioni e omicidi il cui movente principale sembra essere economico, possono celare, invece, dinamiche indicanti la natura sessuale dei delitti. Revitch (1957) riporta il caso di un uomo che aggredisce una donna con un blackjack e che successivamente viene arrestato con l'accusa di aggressione e tentato furto. A una valutazione più attenta, emerse come il soggetto avesse fantasie di legare gli arti inferiori di persone di sesso femminile. Tali fantasie potrebbero avere innescato la dinamica violenta. La polizia, infatti, soffermandosi sulla dichiarazione rilasciata in principio dall'uomo ignorò la presenza nel bagagliaio dell'auto di proprietà dello stesso di un cappio, di un fucile e di una copia del testo di Krafft-Ebing, Psychopathia Sexualis. Revitch concluse che l'erezione, l'eiaculazione e il rapporto sessuale non accompagnano necessariamente un'aggressione o un omicidio violento (a sfondo sessuale), poiché l'aggressione brutale può sostituire l'atto sessuale.

Problema 4. Grubin (1994) elenca una serie di modi in cui un omicidio e un reato sessuale possono essere correlati. Un molestatore sessuale può uccidere la vittima:

- per mettere a tacere l'unico potenziale testimone d'accusa;

- perché essa oppone strenua resistenza;

- accidentalmente, perché preso dal panico;

- partecipando a uno stupro, a causa della pressione psicologica esercitata dal gruppo.

Sebbene in questi casi la violenza omicida e la componente sessuale siano strettamente connesse, i delitti descritti, secondo Grubin, non possono essere considerati omicidi sessuali. Meloy (2000), per esempio, distingue l'omicidio sessuale dall'omicidio associato all'attività sessuale. L'autore individua due tipologie cliniche di assassini sessuali:

1. gli assassini sessuali compulsivi aventi alle spalle una carriera nella criminalità organizzata (solitamente affetti da disturbo antisociale/narcisistico di personalità o da sadismo sessuale; emotivamente distaccati, psicopatici primari, iporeattivi, esenti da esperienze traumatiche precoci);

2. gli assassini sessuali catatimici aventi alle spalle una carriera nella criminalità disorganizzata (solitamente affetti da disturbo dell'umore e da vari disturbi di personalità che possono includere tratti schizoidi ed evitanti; affamati di attaccamento, moderatamente psicopatici, iperreattivi, vittime di traumi infantili fisici e/o sessuali).

Problema 5. Nel 2017 negli Stati Uniti sono stati commessi circa 1.247.321 reati violenti, di cui 17.284 sono omicidi (Uniform Crime Reporting, FBI). Nella categoria "crimine violento" UCR, però, rientrano unicamente le fattispecie delittuose di omicidio e omicidio non colposo; non compare alcuna sottospecificazione indicante la natura degli omicidi perpetrati. Il Canada, invece, fornisce statistiche ufficiali riguardanti il tasso annuo di omicidi sessuali, sebbene la modalità di classificazione dei dati presenti un limite endogeno (Ressler \& Schachtman, 1992). Secondo i parametri classificatori utilizzati dal Canadian
Centre for Justice Statistics la categoria di omicidio sessuale comprende ogni omicidio verificatosi durante la commissione di un reato sessuale. Tuttavia, questa classificazione esclude numerosi casi di omicidio sessuale che però non sono correlati alla commissione di un reato sessuale (per es.: i casi di strangolamento o di ferite da taglio). La mancanza di dati statistici ufficiali a livello nazionale o locale volti a registrare l'incidenza dell'omicidio sessuale unitamente ai problemi classificatori descritti da Grubin ostacolano, pertanto, sia la ricerca scientifica sia la comprensione di questa tipologia omicidiaria.

Problema 6. Dietz (1986) sottolinea come la scarsità di articoli scientifici sull'omicidio sessuale (seriale) sia in gran parte attribuibile al fatto che il fenomeno occorre raramente. Inoltre, gli autori di omicidi a sfondo sessuale non sono giuridicamente classificati come assassini sessuali, per cui la loro identificazione a fini di ricerca diventa ancora più difficile. Per esempio, tra la popolazione carceraria è possibile identificare il numero esatto di stupratori o di pedofili, così come il numero esatto di assassini. Ma è impossibile per un ricercatore disporre del numero esatto di detenuti resisi responsabili di omicidi sessuali presenti sul territorio nazionale, perché essi sono classificati come assassini, non come assassini sessuali. Inoltre, gli assassini sessuali ricorrono a massicce tecniche di negazione e minimizzazione, manifestando la tendenza a manipolare, dominare e controllare gli altri, compresi i ricercatori e i clinici (Douglas \& Olshaker, 1995). La mancanza di collaborazione interdisciplinare è un altro ostacolo pratico alla comprensione dell'omicida sessuale. Spesso criminologi e sociologi studiano il fenomeno criminale da una prospettiva statistico-epidemiologica. Gli investigatori analizzano le informazioni relative al crimine che facilitano la raccolta degli indizi sul luogo del reato, le trascrizioni di dichiarazioni testimoniali, i referti autoptici, il comportamento criminale in base alla scena del crimine. Psichiatri e psicologi si occupano dello stato mentale dell'autore del reato al momento della commissione del delitto. Poiché l'omicidio sessuale è un fenomeno multidimensionale, la mancanza di collaborazione interdisciplinare riduce la comprensione di questa tipologia omicidiaria.

\section{I modelli teorici}

Sotto il profilo teorico l'interesse per l'omicidio sessuale fa riferimento a quattro modelli interpretativi (Purcell \& Arrigo, 2006):

1. il modello del condizionamento classico. Elaborato da MacCulloch e colleghi (1983), sottolinea l'importanza della fantasia sessuale come precursore del comportamento sadicoaggressivo. Lo studio indaga il rapporto tra fantasie violente e passaggio all'atto criminale in un campione costituito da 16 pazienti psichiatrici ospedalizzati, affetti da psicopatia, resisi responsabili di omicidi a sfondo sadico-sessuale. Dallo studio emerge come nella fase ante delictum la totalità di comportamenti compulsivi messi in atto dai soggetti esaminati rappresenti l'attuazione di pattern di fantasie violente e sessualizzate derivanti da un condizionamento classico od operante che collega l'eccitazione sessuale a specifiche immagini mentali stimolanti. Durante le fantasie le immagini di natura sadico-sessuale provocherebbero una eccitazione sessuale precedente l'orgasmo talmente intensa da indurre i soggetti a ricercare in maniera compulsiva il piacere associato a tale eccitazione. 13 dei 16 soggetti esaminati presentavano nella c.d. "fase aurorale" (Norris, 1988) fantasie masturbatorie di intonazione sadica, caratterizzate da crescente intensità ed 
elevata ripetitività. I risultati dimostrano che le fantasie di tipo sadico-sessuale tendono a tradursi in "prove comportamentali" (MacCulloch et al., 1983);

2. il modello motivazionale. Elaborato da Burgess e colleghi (1986), esamina i fattori motivazionali che sottendono l'omicidio sessuale e la violenza sadica. Lo studio, condotto su un campione di 36 assassini sessuali, tutti maschi, quasi tutti bianchi (33 su 36), dotati di buone risorse cognitive, provenienti da nuclei familiari apparentemente normotipici, di condizione economica agiata o per lo meno nella media, evidenzia cinque componenti motivazionali interconnesse:

- l'ambiente sociale inadeguato. I soggetti esaminati mostravano stili di attaccamento negativi sperimentati nella loro vita infantile con le figure d'attaccamento primarie (figure inadeguate disfunzionali, instabili, affette da problemi psichiatrici, propense alla devianza e alla criminalità);

- gli eventi formativi. I traumi "normativi" (la malattia, il divorzio, la morte), i traumi "non-normativi" (fisici, psicologici o sessuali), il fallimento dello sviluppo socioemozionale e il conflitto intrapsichico influenzano negativamente lo sviluppo infantile e adolescenziale di un individuo;

- $\quad$ i tratti critici della personalità, le mappe e i processi cognitivi. L'isolamento sociale conduce il bambino a utilizzare la fantasia come sostituto di positive relazioni e legami sociali, agevolando lo sviluppo in età adulta di una struttura di personalità eccessivamente dipendente dall'attività fantasmatica e da specifici temi dominanti a essa correlati a scapito di qualsiasi interazione sociale di routine o sana) (Burgess et al., 1986). I soggetti esaminati manifestano tratti di personalità critici (isolamento sociale, preferenza per l'autoerotismo, comportamento feticista, tendenza alla ribellione, presenza di aggressività, ricorso alla menzogna cronica, tendenza all'auto-legittimazione). Nei soggetti esaminati la mappatura, intesa come elaborazione cognitiva, avrebbe prodotto i cosiddetti "sogni a occhi aperti", incubi, pensieri accompagnati da vivide componenti visive, fantasie caratterizzate da temi dominanti (potere, controllo, dominio, vendetta, violenza, mutilazione, stupro, tortura, morte) (Purcell \& Arrigo, 2006).

- le azioni verso sé stessi e verso gli altri. I modelli di comportamento attuati dagli assassini sessuali esaminati riflettono una realtà psichica interiore caratterizzata dalla presenza di pensieri disturbanti, privi di gioia, implicanti l'esercizio del dominio sugli altri. Nell'infanzia questi pensieri si traducono in giochi a connotazione negativa, maltrattamento di animali, azioni incendiarie, vandalismo, mancanza di rispetto per gli altri; in adolescenza e in età adulta questi modelli comportamentali disfunzionali pervengono ad agiti sempre più severi e brutali, come la commissione di reati appropriativi e reati violenti) (Burgess et al., 1986, pag. 266).

- i filtri del feedback. Circa i comportamenti agiti in passato, i 36 soggetti tendono a giustificare questi ultimi ricorrendo a "correzioni mentali volte a preservare e proteggere i loro mondi interiori fantasmatici” (Purcell \& Arrigo, 2006, pag. 44).

3. il modello trauma-controllo. Elaborato da Hickey (1997, 2001), spiega il fenomeno dell'omicidio seriale focalizzando l'attenzione sugli eventi traumatici subiti nell'infanzia dagli assassini seriali. Le conseguenze cui gli eventi traumatici esperiti sembrano condurre sono sei:
- l'insorgenza di sentimenti interni negativi;

- la tendenza a rifugiarsi in elaborazioni fantastiche per avere gratificazioni sostitutive;

- $\quad$ il disturbo dissociativo di identità;

- le esperienze di rinforzo del trauma;

- il ritiro psichico difensivo;

- il comportamento omicida.

Inoltre, si rivelano determinanti per il futuro comportamento omicida:

- $\quad$ i fattori predisponenti (biologici, psicologici, sociologici o una combinazione di questi elementi);

- $\quad$ i fattori facilitanti (uso di alcol e droghe, consumo di materiale pornografico, interesse per il mistico e l'occulto).

4. il modello integrato. Sviluppato da Purcell e Arrigo (2006, pp. 53-65), sottolinea l'importanza della parafilia come "sistema di comportamento" (ivi, pag. 53) rintracciabile nell'eziologia dell'erotofonofilia $3 / 4$ l'FBI definisce e classifica la erotofonofilia come una serie di omicidi (più di tre vittime), intervallati da pause, che indicano una premeditazione del reato (Simon, 1997). Gli autori individuano quattro componenti in grado di spiegare il modo in cui fantasie sempre più violente, quando non si dimostano più sufficienti a provocare eccitazione e/o gratificazione sessuale, sfociano in outcome letali, come il "Lust murder":

- lo sviluppo formativo. I fattori biologici, psicologici e sociali uniti a episodi traumatizzanti sono in grado di innescare comportamenti parafiliaci spesso culminanti in agiti erotico-sadici, aggressivi e, in casi estremi, omicidiari (Purcell e Arrigo, 2006, pag. 58);

- lo stato di bassa autostima. I bambini vittime di eventi traumatici sono caratterizzati da un sentimento di autostima bassa che li condizionerà per tutta la vita, interferendo con la capacità di creare legami positivi e catalizzando risposte adattive di tipo fantasmatico volte a ottenere gratificazione sostitutiva, a sfogare la rabbia e l'ostilità sedimentate e ad allentare la tensione psichica;

- lo sviluppo di fantasie sessualizzate precoci attiva il sistema parafiliaco che, gradualmente, diviene un processo funzionante in sé e per sé. L'attività fantasmatica intrusiva, la masturbazione compulsiva, i fattori facilitanti e gli stimoli parafiliaci (per es.: feticci, oggetti insoliti, rituali sadici ed erotici) rappresentano elementi che coadiuvano e sostengono il processo parafiliaco (ibidem);

- lo sviluppo di parafilie tende a subentrare in concomitanza con l'emergere di sintomi intrusivi associati all'evento traumatico subito nell'infanzia (isolamento sociale, masturbazione compulsiva, fantasie sessualizzate precoci).

Con le componenti eziologiche interagiscono, infine, tre elementi:

- le fantasie e gli stimoli di natura parafiliaca;

- il processo di condizionamento orgasmico. La stimolazione genitale compulsiva assoggetta l'individuo alla fantasia parafiliaca sessualmente violenta al punto da risultare indispensabile sia per provocare l'eccitazione sia per raggiungere l'orgasmo;

- gli elementi facilitanti. Il soggetto parafiliaco è ancorato a un sistema di dipendenza (alcol, droghe, pornografia) desensibilizzante che può condurre lo stesso ad agire $\mathrm{i}$ contenuti operativi delle proprie fantasie, commettendo atti di violenza sadica e, in escalazione, di violenza omicida (ivi, 61). 


\section{Il caso di Giovanni Badalotti}

Il profilo. ${ }^{1}$ Giovanni Badalotti inizia la propria carriera delinquenziale in età preadolescenziale manifestando comportamenti antisociali e aggressivi che perdurano con la transizione all'età adulta. Parametri descrittivi quali l'incidenza (il numero di reati commessi), l'onset delinquenziale precoce (12 anni), la continuità (l'attività delinquenziale del soggetto si protrae lungo un arco temporale di trent'anni), il termine (20/03/2011, data della decorrenza della pena inflitta), la specializzazione (la tendenza a commettere reati appropriativi), l'escalation (il passaggio da reati appropriativi, senza interazione, a reati di detenzione e spaccio di sostanze stupefacenti fino a reati contro la persona, nello specifico i reati di violenza sessuale e di omicidio) indicano una carriera criminale strutturata, caratterizzata da una progressiva accelerazione delle cariche violente e aggressive.

Significative a tale proposito le risultanze di due perizie psichiatriche cui il soggetto è stato sottoposto, l'una nel corso delle indagini preliminari (nelle forme dell'incidente probatorio), l'altra in sede di giudizio abbreviato, dalle quali emerge il profilo di un soggetto affetto da "Disturbo di Personalità Antisociale", caratterizzato da "egoismo negativo" che "agisce in maniera violenta e attua comportamenti pericolosi per avvantaggiarsi occasionalmente" (sentenza n. 225/12 del 31/05/2012, emessa dal Tribunale di Piacenza). Sotto il profilo criminogenico rilevano i seguenti elementi: il soggetto cresce sperimentando condizioni di degrado familiare, sociale e morale: un padre analfabeta, dedito all'alcol, incapace di assolvere ai compiti genitoriali che ha abusato sessualmente delle proprie figlie; una madre severa, dedita all'alcol e alla prostituzione, inadeguata sotto il profilo educativo; dei fratelli vessanti e violenti, verso i quali il soggetto serba profondo rancore. In un simile contesto di deprivazione familiare, affettiva, educativa e culturale Badalotti sembra avere sviluppato una personalità schiva, difensiva, unicamente centrata sul soddisfacimento di bisogni primari, incapace di tollerare le frustrazioni o l'esposizione a vicende personali stressanti.

In relazione al reato di violenza sessuale, nella forma tentata del delitto, il soggetto mostra un atteggiamento marcatamente denegante. Ricostruisce con controllato distacco la dinamica omicidiaria, offrendo motivazioni esplicative del disegno criminoso assai laconiche (rapporti di vicinato tesi). Rileva la totale assenza di sentimenti di colpa o rimorso per le condotte antigiuridiche poste in essere.

Il fatto-reato. Castelvetro Piacentino (PC), 20 marzo 2011. Giovanni Badalotti, 42enne, uccide Stella Paroni, 91 anni, gettandola dal balcone del terzo piano della palazzina in cui l'anziana vive sola. Attorno alle 4 del mattino l'uomo stacca la corrente dal contatore dell'abitazione della vittima, costringendola a scendere in cantina per riattivarlo. La donna lascia la porta aperta. Badalotti si intrufola nell'abitazione. Quando l'anziana rientra, l'uomo la aggredisce, la denuda e tenta di violentarla. In seguito, scaraventa la donna dal terrazzo, scende in cortile, carica il corpo esanime su un carretto e, infine, lo trasporta in un canale accanto a una discarica a circa cinquecento metri dalla palazzina. Poche ore dopo Badalotti viene arrestato con le accuse di omicidio volontario, tentata violenza sessuale e occultamento di cadavere.

La prospettiva situazionale. Sebbene l'evidenza empirica dimostri che alcuni assassini sessuali uccidono in quanto affetti da psicopatologia o da gravi problemi di gestione della rabbia, esistono prove a sostegno della tesi secondo cui l'omicidio sessuale rappresenti un possibile esito di un atto di aggressione sessuale (Beech et al., 2005; Beech et al., 2006). Risultati emergenti da ricerche empiriche recenti (Mieczkowski e Beauregard, 2010;
Beauregard \& Mieczkowski, 2012) indicano come tra gli assassini sessuali e gli aggressori sessuali non si ravvisi una differenza qualitativa: ciò che sembra differenziare l'omicidio sessuale dall'aggressione sessuale è la presenza di fattori situazionali anziché di fattori costituzionali. Lo studio condotto da Mieczkowski e Beauregard (2010) indica come la probabilità che una aggressione sessuale si trasformi in un omicidio sia condizionata dalla combinazione di caratteristiche della vittima, caratteristiche situazionali e caratteristiche della scena del crimine. La più letale delle combinazioni configura il seguente scenario: durante l'aggressione il soggetto utilizza un'arma, non porta a compimento l'atto predatorio pianificato, trascorre un lasso temporale superiore a 30 minuti con la vittima (Chan \& Heide, 2009). Questo studio evidenzia come l'incapacità di agire il comportamento sessualmente violento pianificato in origine dall'aggressore - nonostante il lungo lasso di tempo intercorso a contatto con la vittima - scateni una conseguente reazione di rabbia facilitante l'acting out omicidiario. Tale epilogo può essere facilitato dalla presenza di un'arma durante l'evento vittimizzatorio. La prospettiva delineata da Healey e colleghi (2016) fa emergere la figura del c.d. "Situational Precipitated Offender", cioè un aggressore di tipo "situazionale" il cui obiettivo primario è la perpetrazione di un atto di violenza sessuale a danno della vittima designata. La presenza di elementi situazionali caratterizzanti l'evento predatorio (per es.: lo stato di intossicazione da sostanze alcoliche o sostanze stupefacenti dell'aggressore, la resistenza che la vittima oppone alla volontà dell'aggressore, la tipologia di relazione tra vittima e aggressore, la presenza di un'arma) possono divenire "fattori precipitanti" capaci di attivare la suscettibilità dell'aggressore contribuendo all'esternazione di improvvise esplosioni di violenza omicidiaria. La prospettiva criminologica appena esposta potrebbe essere applicata al caso di Giovanni Badalotti. Il delitto di violenza sessuale nella forma tentata ${ }^{2}$ commesso da Badalotti a danno di Stella Paroni appare compatibile con l'agito omicidiario che ha caratterizzato la fase epilogativa dell'evento vittimizzatorio. Il mancato compimento dell'azione sessuale violenta pianificata dall'aggressore - elemento disturbante che ha impedito la scarica della pulsione aggressiva del soggetto, influenzando le successive fasi dell'evento predatorio - potrebbe essersi tradotto in un fattore situazionale precipitante, innescando un'escalation di aggressività risoltasi nell'omicidio dell'anziana. La Corte d'Assise di Bologna, infatti, ha riconosciuto il movente sessuale dell'omicidio; movente che rende inapplicabili le aggravanti della premeditazione e dei motivi abbietti e futili (tesi confermata dalla Corte di Cassazione). Badalotti, pertanto, sembra aderire al profilo del "Situational Precipitated Offender". Questa tipologia di aggressore, a differenza del "Lust killer", il quale premedita l'omicidio della vittima prescelta (in genere sconosciuta), perché trae piacere dall'atto di uccidere, sceglie vittime conosciute, mostra elementi di premeditazione connessi all'esecuzione di un atto di violenza sessuale piuttosto che alla pianificazione di un disegno omicidiario, non presentando i segni manifesti della rabbia durante la fase iniziale dell'evento predatorio. La rabbia esplode a causa della resistenza della vittima. Questa rabbia crescente, unita alla pre-

Osservazione criminologica effettuata dalla scrivente durante l'attività di osservazione e trattamento espletata ai sensi dell'art. 80 O.P

2 Articolo 56 Codice Penale. Il delitto tentato, contrapposto al delitto consumato, indica in diritto penale un delitto che non è giunto alla sua consumazione perché non si è verificato l'evento voluto dal reo o perché, per ragioni indipendenti dalla sua volontà, l'azione non è comunque giunta a compimento. 
senza di un'arma e all'uso di sostanze stupefacenti da parte dell'aggressore, può rivelarsi un fattore scatenante l'escalation omicidiaria (Healey et al., 2016, pag. 526).

\section{Conclusioni}

Le considerazioni svolte in questo contributo hanno posto in evidenza come l'omicidio sessuale rappresenti un evento delittuoso assai complesso. Essendo la produzione scientifica in materia di omicidio sessuale lacunosa, si è tentato di rintracciare le ragioni di questo vuoto empirico.

Si è posto l'accento sul problema derivante dalla eterogeneità terminologica impiegata dalla comunità scientifica internazionale per descrivere la violenza omicida a sfondo sessuale, sulle difficoltà di analisi della scena del crimine durante la fase investigativa, sugli aspetti confusivi emergenti dalla differenziazione introdotta in ambito criminologico tra "omicidio sessuale" e "omicidio associato a comportamento sessuale", sull'assenza di dati statistici ufficiali a livello nazionale o locale volti a registrare l'incidenza dell'omicidio sessuale e, infine, sul mancato o carente lavoro interdisciplinare durante lo svolgimento dell'attività di ricerca.

Si è proceduto a descrivere i principali modelli teorici elaborati per spiegare l'omicidio sessuale e, poi, ad analizzare un caso di cronaca nera italiana secondo la prospettiva situazionale. I risultati di questo studio sottolineano come l'omicidio sessuale sia un crimine dinamico verso cui le aggressioni sessuali, in presenza di un comportamento di escalation aggressiva elicitato da specifici fattori situazionali, possono culminare. Al centro di questa prospettiva c'è un'attenzione rivolta ai fattori precipitanti che possono modificare il disegno criminoso originariamente pianificato dall'aggressore, scatenando così la violenza omicida.

\section{Bibliografia}

Beauregard E., Mieczkowski T. (2012), "Risk estimations of the conjunction of victim and crime event characteristics on the lethal outcome of sexual assaults", Violence and Victims, 27:470-86.

Beech A. R., Oliver C., Fisher D., Beckett R. (2006), STEP 4: The sex offender treatment programme in prison: Addressing the offending behaviour of rapists and sexual murders, The Centre for Forensic and Family Psychology, Birmingham, UK.

Beech A., Fisher D., Ward T. (2005), "Sexual murderers' implicit theories", Journal of Interpersonal Violence, 20:1366-89.

Berkowitz L. (1989), "Frustration-aggression hypothesis. Examination and reformulation", Psychol Bull, 106:59-73.

Brittain R.P. (1970), “The sadistic murderer", Medicine, Science, and Law, 10: 98.

Burgess A.W., Hartman, C.R., Ressler, R.K., Douglas, J.E., McCormack, A. (1986), Sexual homicide: A motivational model, Journal of Interpersonal Violence, 1:151-272.

Chan H.C., Heide K.M. (2009), "Sexual homicide: A synthesis of the literature", Trauma, Violence, \& Abuse, 10:31-54.

De River J.P. (1958), Crime and the sexual psychopath, Charles C Thomas, Springfield, IL.

Dietz P.E. (1986), "Mass, serial, and sensational homicides", Bulletin of the New York Academy of Medicine, 62:477-91.

Douglas J.E., Olshaker M. (1995), Mind hunter, Pocket Books, New York.

Grubin D. (1994), "Sexual murder", British Journal of Psychiatry, 165:624-9.
Hazelwood R.R., Douglas J.E. (1980), “The lust murderer”, FBI Law Enforcement Bulletin, 49:1-5.

Healey J., Beauregard E., Beech A., Vettor S. (2016), "Is the sexual murderer a unique type of offender? A typology of violent sexual offenders using crime scene behaviors", Sexual Abuse: A Journal of Research and Treatment, 28:512-33.

Hickey E. (1997²), Serial murderers and their victims, Wadsworth, Belmont, CA.

Hickey E. $\left(2001^{3}\right)$, Serial murders and their victims, Wadsworth, Belmont, CA.

Hickey E. (2005), Sex crimes and paraphilia, Prentice-Hall, Upper Saddle River.

Krafft-Ebing, R. von (1886), Psychopathia sexualis, Philadelphia: F.A. Davis, Philadelphia.

Lorenz K. (1982), L'aggressività, Mondadori, Milano.

Lucarelli C., Picozzi M. (2015), Serial Killer. Storie di ossessione omicida, Milano, Mondadori.

MacCulloch M.C., Snowden P.J., Wood P., Mills H.E. (1983), "Sadistic fantasy, sadistic behavior, and offending", British Journal of Psychiatry, 143:20-9.

Malmquist, C.P. (1996), Homicide: A psychiatric perspective, American Psychiatric Press, Washington, DC.

Meloy J.R. (2000), "The nature and dynamics of sexual homicide: An integrative review", Aggression and Violent Behavior, 5:1-22.

Menninger, K. (1963), The vital balance, Viking Press, New York. Merzagora Betsos I. (2006), Criminologia della violenza e dell'omicidio dei reati sessuali dei fenomeni di dipendenza, CEDAM, Padova.

Mieczkowski T., Beauregard E. (2010), "Lethal outcome in sexual assault events: A conjunctive analysis", Justice Quarterly, 27:332-61.

Money J. (1990), "Forensic sexology. Paraphiliac serial rape (biastophilia) and lust murder (erotophonophilia)", American Journal of Psychotherapy, 44:26-36.

Norris, J. (1988), Serial Killers, Anchor Books, New York.

Pais S., Perrotta G. (2015), L'indagine investigativa. Manuale teorico-pratico. Primiceri Editore, Padova.

Palermo G.B. (2010), Severe personality-disordered defendants and the insanity plea in the United States. A proposal for Change. Boom Juridisch Uitgevers, The Hague, The Netherlands.

Palermo G.B., Mastronardi V.M. (2005), Il profilo criminologico. Dalla scena del crimine ai profili psicologici. Giuffrè Editore, Milano.

Palermo, G.B., Mastronardi V. (2012), “L'omicidio. Profili comparatistici Italia-USA", Rivista di Psichiatria 47:1-10.

Prentky R.A., Burgess A.W., Rokous R., Lee A., Hartman C., Ressler R.K., Douglas J. (1989), The presumptive role of fantasy in serial sexual homicide, American Journal of Psychiatry, 146:887-91.

Purcell C.E., Arrigo B. A. (2006), The psychology of lust murder. Paraphilia, sexual killing, and serial homicide, Academic Press, Burlinghton, MA.

Raine A., (2016), L'anatomia della violenza. Le radici biologiche del crimine, Mondadori Università, Milano.

Ressler R.K., Burgess A.W., Douglas J.E. (1988), Sexual homicide: Patterns and motives, Free Press, New York

Ressler R.K., Schachtman T. (1992), Whoever fights monsters, St. Martin's Press, New York.

Revitch E. (1957), "Sex murder and sex aggression", Journal of the Medical Society of New Jersey, 54:519-24.

Rossi L., Zappalà A. (2005), Personalità e crimine. Elementi di psicologia criminale, Carocci, Roma. 
Schlesinger L.B. (2004), Sexual murder: Catathymic and compulsive Homicides, CRC Press, Boca Raton.

Schlesinger L.B., Revitch, E. (19972), Sexual dynamics of antisocial behavior, Charles $\mathrm{C}$ Thomas, Springfield.

Simon R.J. (1997), I buoni lo sognano, i cattivi lo fanno.
Psicopatici, stupratori, serial killer. Raffello Cortina Editore, Milano.

Sutherland E.H., Cressey D.R. (1959), Principles of criminology. Lippincott, Chicago.

Wolfgang M., Ferracuti F. (1967), The subculture of violence. Social Science Paperbacks, London.
Correspondence: Francesca Vitale.

E-mail: chiccavitale@gmail.com

Key words: sexual homicide, problem issues, theoretical models, case analysis.

Parole chiave: omicidio sessuale, aspetti problematici, modelli teorici, analisi di un caso.

Palabras clave: homicidio sexual, aspectos problemáticos, modelos teóricos, análisis de un caso.

Received for publication: 9 March 2020.

Accepted for publication: 30 April 2020.

This article is distributed under the terms of the Creative Commons Attribution Noncommercial License (by-nc 4.0) which permits any noncommercial use, distribution, and reproduction in any medium, provided the original author(s) and source are credited.

${ }^{\circ}$ Copyright: the Author(s), 2020

Licensee PAGEPress, Italy

Rivista di Psicopatologia Forense, Medicina Legale, Criminologia 2020; $25: 269$

doi:10.4081/psyco.2020.269 